\title{
THE CONNECTIVE $K$-THEORY OF SPINOR GROUPS
}

\author{
LUÍSA MaGalHães
}

\begin{abstract}
We study the properties of the connective $K$-theory with $\boldsymbol{Z}_{2}$ coefficients of the Lie groups $S p i n(n)$. This generalises some work of L. Hodgkin.
\end{abstract}

\section{o. Introduction}

In a previous paper [4] we obtained the Hopf algebra structure of $k^{*}(G ; Q(P))$ where $G$ is a compact connected Lie group and $Q(P)$ is the quotient ring of $\boldsymbol{Z}$ with respect to a multiplicative subset generated by a set of primes, such that $H^{*}(G ; Q(P))$ is totsion free.

Here we study the properties of the connective $K$-theory of the Lie groups $\operatorname{Spin}(n)$. Since $H^{*}(\operatorname{Spin}(n) ; \boldsymbol{Z})$ is torsion free if $n \leq 6$, we are only interested in the cases where $n \geq 7$. We will see that $k^{*}(\operatorname{Spin}(n))$ has only two torsion and we will give the algebra structure of $k^{*}\left(\operatorname{Spin}(n) ; \mathbf{Z}_{2}\right) /\left\{x \in k^{*}\left(\operatorname{Spin}(n) ; \mathbf{Z}_{2}\right)\right.$ : $\left.t^{-1} x=0\right)$ for $n \geq 7\left(t^{-1}\right.$ is the canonical generator of $\left.k^{*}(p t)\right)$.

Our paper generalises part of earlier work of L. Hodgkin, who computed the usual $K$-theory of compact Lie groups [3].

\section{Preliminaries}

Let $k$ and $K$ denote, respectively, the spectrum for connective $K$-theory and the usual $K$-theory. There is a natural map of ring spectra $j: k \rightarrow K$. We recall that $k^{*}(p t)=\mathbf{Z}\left[t, t^{-1}\right]$, the Laurent polynonial ring generated by the reduced Hopf bundle $t^{-1} \in K^{-2}(p t)$ and its inverse, and that $k^{*}(p t)=Z\left[t^{-1}\right]$. We denote by $m: k \rightarrow k$ the morphism of spectra corresponding to multiplication by $t^{-1}$.

If we introduce $\mathbf{Z}_{p}$ coefficients in the $k$-cohomology we still obtain an associative multiplication in $k^{*}\left(; \mathbf{Z}_{p}\right)$.

Let $H Z$ be the Eilenberg-MacLane spectrum with integer coefficients. There is a map of ring spectra $n: k \rightarrow H Z$ such that it induces the homomorphism $n^{*}: k^{*}(p t) \rightarrow H^{*}(p t ; \mathbf{Z})$ given by $n^{*}\left(a t^{-n}\right)=\left\{\begin{array}{ll}a & \text { if } n=0 \\ 0 & \text { if } n>0\end{array}, a \in \mathbf{Z}, n \in \mathbf{N} \cup\{0\}[5]\right.$.

For each $C W$ complex $X$ and each prime $p$ there is an exact sequence

$$
0 \longrightarrow \mathbf{Z}_{p} \otimes k^{*}\left(X ; \mathbf{Z}_{p}\right) \stackrel{\ddot{n}^{*}}{\longrightarrow} H^{*}\left(X ; \mathbf{Z}_{p}\right) \longrightarrow \operatorname{Tor}_{1, *}^{\mathbf{Z}_{p}\left[t^{-1}\right]}\left(\mathbf{Z}_{p} ; k^{*}\left(X ; \mathbf{Z}_{p}\right), \longrightarrow 0\right.
$$

where $\tilde{n}^{*}$ is $1 \otimes n^{*}: \mathbf{Z} \otimes k^{*}(X) \rightarrow \mathbf{Z} \otimes H^{*}(X ; \mathbf{Z})$ reduced $\bmod p[4]$. 


\section{Atiyah-Hirzebruch spectral sequence}

Let $X$ be a compact $C W$ complex. We are going to consider the following Atiyah- Hirzebruch spectral sequences: $\left(E_{r}^{* *}, d_{r}\right)_{r \geq 2}$ converging to $K^{*}(X)$ and $\left({ }^{\prime} E_{r}^{* *}, d_{r}^{\prime}\right)_{r \geq 2}$ converging to $k^{*}(X)$. Let $F_{p}^{m}=\operatorname{ker}\left(K^{m}(X) \rightarrow K^{m}\left(X^{p-1}\right)\right)$ and ${ }^{\prime} F_{p}^{m}=\operatorname{ker}\left(k^{m}(X) \rightarrow k^{m}\left(X^{p-1}\right)\right)$ be the filtrations. The first spectral sequence is compatible with the Bott isomorphism.

We note that, since $K^{q}(p t)=0=k^{q}(p t)$ if $q$ is odd and $k^{q}(p t)=0$ if $q>0$, then: ' $E_{r}^{p, q}=0=E_{r}^{p, q}$ for all $p \in \mathbf{Z}, r \geq 2, q$ an odd integer; ' $E_{r}^{p, q}=0$ if $q>0$; and all the differentials of even degree are zero. Moreover, we have for ali $i, n \in \mathbf{Z}: F_{n-1}^{i}=F_{n}^{i}$ and ${ }^{\prime} F_{n-1}^{i}={ }^{\prime} F_{n}^{i}$ if $n-i$ is even; $F_{n}^{i}=F_{n+1}^{i}$ and $' F_{n}^{i}={ }^{\prime} F_{n+1}^{i}$ if $n-i$ is odd; $m^{*}\left(F_{n}^{i}\right)=F_{n}^{i-2}$ and ${ }^{\prime} F_{n}^{n}=k^{n}(X)$.

The same notations will be used when we introduce $\boldsymbol{Z}_{p}$ coefficients.

\subsection{Proposition. [4]:}

Let $X$ be a compact $C W$ complex. Then:

i) $j_{s}^{* *}:{ }^{\prime} E_{s}^{p, q} \rightarrow E_{s}^{p, q}$ is an isomorphism for $q \leq-\operatorname{dim} X+1$,

ii) $j_{\left.\right|^{*} F_{n}^{m}}^{*}$ is an isomorphism onto $F_{n}^{m}$ for all $m \in \mathbf{Z}$ and $n \geq m+s-1$, if $d_{r}=0$ for $r>s$, where $j_{s}^{* *}$ and $j^{*}$ are the obvious morphisms induced by $j: k \rightarrow K$.

\subsection{Proposition.}

Let $X$ be a $C W$ complex such that $K^{*}(X)$ is torsion free. If the differentials $d_{r}$ in the spectral sequence converging to $K^{*}(X)$ are zero for $r>s$ (we suppose $s$ add since the differentials of even degree are zero), then $\left\{y \in k^{*}(X)\right.$ : $\left.t^{(-s+1) / 2} y=0\right\}=\left\{y \in k^{*}(X): \lambda y=0\right.$ for some $\left.\lambda \in \mathbf{Z}-\{0\}\right\}$.

\section{Proof:}

We consider the spectral sequences $\left(E_{r}^{* *}, d_{r}\right)$ and $\left({ }^{\prime} E_{r}^{* *}, d_{r}^{\prime}\right)$ converging to $K^{*}(X)$ and $k^{*}(X)$, respectively.

We have the following commutative diagram for all $m \in Z, i \geq 0$ :

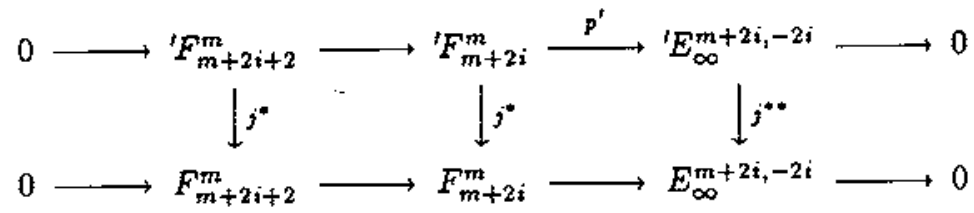

where $j^{*}$ and $j^{* *}$ are the maps induced by $j: k \rightarrow K$ and $p^{\prime}$ is the map of the extension exact sequence.

Let $y \in k^{m}(X)$ be such that $\lambda y=0$ for some $\lambda \in \mathbb{Z}-\{0\}$. Since $j^{*}$ : ${ }^{\prime} F_{m}^{m-s+1} \rightarrow F_{m}^{m+s+1}$ is an isomorphism (by 2.1) and $K^{*}(X)$ is torsion free, then $t^{(-s+1) / 2} y=0$. 
We suppose now that $y \in k^{m}(X)$ and $t^{(-s+1) / 2} y=0$. To finish the proof it is enough to show that $\lambda y \in{ }^{\prime} F_{m+s-1}^{m}$ for some non zero integer $\lambda$ (by 2.1).

Wo are going to prove by induction on $i \geq 0$ that there exists $\lambda \in \mathbb{Z}-\{0\}$ such that $\lambda y \in \in^{\prime} F_{m+2 i+2}^{m}$.

For $i=0$ we have $j^{* *} p^{\prime} y=0$ which implies $p^{\prime} y \in i m d_{s}$. Since all the differentials $d_{r}$ are torsion-valued [1], there exists $\lambda \in \mathbf{Z}-\{0\}$ so that $\lambda p^{\prime} y=0$. Therefore, by the exactness of the extension sequence, $\lambda y \in{ }^{\prime} F_{m+2}^{m}$ as required.

The inductive step is proved in the same way and the result follows.

\subsection{Proposition. [4]:}

Let $X$ be a compact $C W$ complex and let $L$ be equal to $Z$ or $Z_{p}$ (p prime), then $x \in H^{p}(X ; L)$ lies in the image of $n^{*}: k^{*}(X ; L) \rightarrow H^{*}(X ; L)$ if and only if $x$ considered as an element of ' $E_{2}^{p, 0}$ is an infinite cycle in the spectral sequence $\left(' E_{r}^{* *}, d_{r}^{\prime}\right)$ converging to $k^{*}(X ; L)$.

\section{The Lie group $\operatorname{Spin}(n)$}

In [2] Borel proved that:

i) $H^{*}\left(\operatorname{Spin}(n) ; \mathbf{Z}_{2}\right)$ is an algebra with a simple system of generators $x_{i}, x$ where degree $x_{i}=i \in S_{n}=\{i \leq n-1: i$ is not a power of 2$\}$ and degree $x=2^{s(n)-1}, s(n)$ is the integer such that $2^{s(n)-1}<n \leq 2^{s(n)}$. Moreover, for ail $i \in \mathbf{N}$ we have: $S q^{i} x_{j}=\left(\begin{array}{c}j \\ i\end{array}\right) x_{i+j}$ if $j \in S_{n}, i+j \in S_{n}$ and $S q^{i} x_{j}=0$ otherwise; $S q^{i} x=0$.

ii) $H^{*}\left(\operatorname{Spin}(n) ; \mathbf{Z}_{2}\right)$ is torsion free if $n \leq 6$ and is an abelian group isomorphic to the direct sum of copies of $\mathbf{Z}$ and $\mathbf{Z}_{2}$ if $n \geq 7$.

We are interested in the cases where $n \geq 7$.

By the results of [3; III-2] and proposition 2.1 we conclude that in the spectral sequence converging to $k^{*}\left(\operatorname{Spin}(n) ; \mathbf{Z}_{2}\right)$ the only possibily non zero differential is $d_{3}^{\prime}:{ }^{\prime} E_{3}^{m, q} \rightarrow{ }^{\prime} E_{3}^{m+3, q-2}$ and that it coincides with $d_{3}$ when $q<0$. Therefore, we will use the same symbol $d_{3}$ for both.

If we identify $E_{3}^{m, q}$ with $H^{m}\left(\operatorname{Spin}(n) ; \mathbf{Z}_{2}\right)$ when $q$ is even and less or equal to zero, we get $d_{3}=S q^{1} S q^{2}+S q^{2} S q^{1}$ and we have the following relations:

i) $d_{3} x_{i}=\left\{\begin{array}{cc}x_{i+3} & \text { if } \text { is odd, } i+3 \in S_{n} \\ 0 & \text { otherwise }\end{array} ; d_{3} x=0\right.$;

ii) if $2 i \in S_{n}$ then $x_{2 i}=d_{3} x_{2 i-3}$;

iii) $x_{i}^{2}=\left\{\begin{array}{c}x_{2 i} \text { if } 2 i \in S_{n} \\ 0 \text { if } 2 i \notin S_{n}\end{array}\right.$ and $x^{2}=0$.

We are going to describe erd $_{3} / \mathrm{imd}_{3}$. We will use the following notation:

a) $S_{n}^{1}=\left\{i\right.$ odd $\left.\in S_{n} ; i+3 \in S_{n}\right\}, S_{n}^{2}=\left\{i\right.$ odd $\in S_{n}: i+3 \in S_{n}$ and $2 i+6 \notin$ $\left.S_{n}\right\}$ and $S_{n}^{3}=\left\{i\right.$ odd $\in S_{n}: i+3 \in S_{n}$ and $\left.2 i+6 \in S_{n}\right\}$

b) $y_{j}=x_{j} x_{j+3}$ if $j \in S_{n}^{2}$ and $z_{k}=x_{k} x_{k+3}+x_{2 k+6}$ if $k \in S_{n}^{3}$;

c) $\bar{u}$ is the image under the projection kerd $d_{3} \rightarrow k e r d_{3} / i m d_{3}$ of any element $u \in$ kerd $_{3}$. 


\subsection{Proposition.}

Using the above notation, kerd $d_{3} /$ imd $_{3}$ is a $\mathbf{Z}_{2}$ exterior algebra generated by $\left(x_{i}\right)_{i \in S_{n}^{1}}, \bar{x},\left(\bar{y}_{j}\right)_{j \in S_{n}^{2}}$ and $\left(\bar{z}_{k}\right)_{k \in S_{n}^{3}}$.

\section{Proof:}

We consider the differential algebra $A=\left(H^{*}\left(\operatorname{Spin}(n) ; \mathbb{Z}_{2}\right), d_{3}\right)$. First we note that it is enough to prove that $H_{*}(A /(x))$ is an exterior algebra on the generators $\left(\bar{x}_{i}\right)_{i \in S_{n}^{1}},\left(\bar{y}_{j}\right)_{j \in S_{n}^{2}}$ and $\left(\bar{z}_{k}\right)_{k \in S_{n}^{3}}((x)$ denotes the ideal generated by $x)$.

Let $B_{n}$ denote $H^{*}\left(\operatorname{Spin}(n) ; \mathbb{Z}_{2}\right) /(x)$. We are going to prove the result by induction on $n \geq 6$. We note that $B_{n}$ is isomorphic to a subalgebra of $H^{*}\left(\operatorname{Spin}(n) ; \mathbf{Z}_{2}\right)$. We shall therefore use the same notation for the corresponding generators of both algebras.

The inductive hypothesis is obviously true for $n=6$.

We have three different cases to consider:

i) $n-1$ is a power of 2 . Therefore $B_{n}=B_{n-1}$.

ii) $n-1$ is odd. Therefore $B_{n}$ has one more generator, $x_{n-1}$, than $B_{n-1}$. Since $d_{3} x_{n-1}=0$ and $x_{n-1} \notin i m d_{3}$ the result follows.

iii) $n-1$ is even and it is not a power of 2 . Thus $B_{n}$ has one more generator, $x_{n-1}$ than $B_{n-1}$ and $x_{n-1}=d_{3} x_{n-4}$.

We consider now the following exact sequence:

$$
0 \longrightarrow\left(x_{n-1}\right) \stackrel{i}{\longrightarrow} B_{n} \stackrel{p}{\longrightarrow} B_{n} /\left(x_{n-1}\right) \longrightarrow 0
$$

where $i$ is the inclusion and $p$ is the projection. It induces the exact triangle:

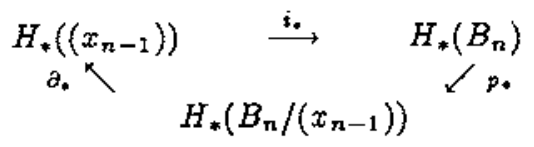

We can replace $B_{n} /\left(x_{n-1}\right)$ by $B_{n-1}$ because they are isomorphic as differential algebras. Clearly, $d_{3} x_{i}=0$ if $i \in S_{n}^{1}, d_{3} y_{j}=x_{j+3}^{2}=0$ if $j \in S_{n}^{2}$ and $d_{3} z_{k}=x_{k+3}^{2}+x_{2 k+6}=0$ if $k \in S_{n}^{3}$.

We have to distinguish two subcases:

a) $\frac{n-1}{2}$ is even. Here $S_{n}^{1}=S_{n-1}^{1} \backslash\{n-4\}, S_{n}^{2}=\left(S_{n-1}^{2} \backslash\{r\} \cup\{n-4\}\right.$ and $S_{n}^{3}=S_{n-1}^{3} \cup\{r\}$, where $r=\frac{n-7}{2}$.

By induction, we have $H_{*} B_{n-1}=\Lambda_{\mathbf{Z}_{2}}\left(\left(\bar{x}_{i}\right)_{i \in S_{n-1}^{1}},\left(\bar{y}_{j}\right)_{j \in S_{n-1}^{2}},\left(\bar{z}_{k}\right)_{k \in S_{n-1}^{3}}\right.$. The generators $\left(\bar{x}_{i}\right)_{i \in S_{n}^{2}},\left(\bar{y}_{j}\right)_{j \in S_{n}^{2} \backslash\{n-4\}}\left(\bar{z}_{k}\right)_{k \in S_{n}^{3}}$ belong to $i m p_{*} ; p_{*} \bar{z}_{r}=\bar{y}_{r}$; and $\partial_{4} \bar{x}_{n-4}=\bar{x}_{n-1}$. As $d_{3}\left(a \bar{x}_{n-4}\right)=a \bar{x}_{n-1}$ in $B_{n}$ if $a$ is a cycle, we have that $\partial_{*}\left(\bar{a} \bar{x}_{n-4}\right)=\bar{a} \bar{x}_{n-1}$ if $\bar{a} \in H_{*} B_{n-1}$. Thus $i m p_{*}=\Lambda_{\mathbf{z}_{2}}\left(\left(\bar{x}_{i}\right)_{i \in S_{n}^{2}},\left(\bar{y}_{j}\right)_{j \in S_{n}^{2}}\right.$, $\left.\left(\bar{z}_{k}\right)_{k \in S_{n}^{3}-\{r\}}\right)$. 
We denote by $R$ the algebra $i m p_{*}$. The map $\partial_{*}$ is $R$-linear and as an $R-$ module $H_{*}\left(B_{n-1}\right)$ is free on the two generators 1 and $\bar{x}_{n-4}$. Moreover $\partial_{*} 1=$ $0, \partial_{*} \bar{x}_{n-4}=\bar{x}_{n-1}$ and the map

$$
\begin{aligned}
B_{n-1} \rightarrow\left(x_{n-1}\right) \\
a \rightarrow a x_{n-1}
\end{aligned}
$$

is an isomorphism of differential modules. Therefore $H_{*}\left(\left(x_{n-1}\right)\right) a$ is free $R$ module isomorphic to $R \bar{x}_{n-1} \oplus R \bar{x}_{n-4} \tilde{x}_{n-1}$ and $H_{*} B_{n}$ is isomorphic to $R$. $1 \oplus R \bar{x}_{n-4} \bar{x}_{n-1}$, as a $\mathbf{Z}_{2}$-vector space.

To check that $H_{*} B_{n}$ is an exterior algebra on the given elements, it remains to show that they have zero square, which can be easily verified.

b) $\frac{n-1}{2}$ is odd. The proof is similar to the previous one.

\subsection{Lemma.}

The kernel of $j^{*}: k^{*}\left(\operatorname{Spin}(n) ; \mathbf{Z}_{2}\right) \rightarrow k^{*}\left(\operatorname{Spin}(n) ; \mathbf{Z}_{2}\right)$ is equal to the set $A=\left\{y \in k^{*}\left(\operatorname{Spin}(n) ; Z_{2}\right): t^{-1}=0\right\}$.

Proof:

The inclusion $k e r j^{*} \supset A$ follows from the equality $F_{r}^{r-2}=' F_{r}^{r-2}$ (proposition 2.1). The other inclusion $k e r j^{*} \subset A$ is trivial.

\subsection{Proposition.}

The kernel of $j^{*}: k^{*}\left(\operatorname{Spin}(n) ; \mathbf{Z}_{2}\right) \rightarrow k^{*}\left(\operatorname{Spin}(n) ; \mathbf{Z}_{2}\right)$ is mapped isomorphically onto imd $d_{3}$ by the map $n^{*}: k^{*}\left(\operatorname{Spin}(n) ; \mathbf{Z}_{2}\right) \rightarrow H^{*}\left(\operatorname{Spin}(n) ; \mathbf{Z}_{2}\right)$.

\section{Proof:}

We will show first that $n^{*}\left(\right.$ kerj $\left.j^{*}\right)=i m d_{3}$.

Since ' $E_{\infty}^{i, \theta} \approx k e r d_{3}$ and $E_{\infty}^{i, 0} \approx k e r d_{3} / i m d_{3}$ we have the following commutative diagram:

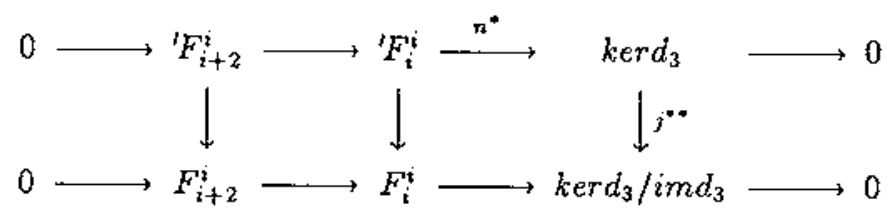

where $j^{* *}$ is the projection. It follows from the exactness of the rows that $n^{*}\left(k e r j^{*}\right)=i m d_{3}$.

It remains to show that $n_{\mid k e r j^{*}}^{*}$ is injective. This is implied by the injectivity of $j_{\mid i m m}^{*}$. (lemma 3.2).

We are now able to describe $k^{*}\left(\operatorname{Spin}(n) ; \mathbf{Z}_{2}\right) / i m j^{*}$. We note that by propositions 2.3 and 3.3 the map $n^{*}$ induces an epimorphism $\bar{n}^{*}: k^{*}\left(\operatorname{Spin}(n) ; \mathbb{Z}_{2}\right)$ 
$/ i m j^{*} \rightarrow k e r d_{3} / i m d_{3}$. Thus we can take elements $\left(\bar{w}_{i}\right)_{i \in S_{n}^{1}},\left(\bar{u}_{j}\right)_{j \in S_{n}^{2}},\left(\bar{v}_{k}\right)_{k \in S_{n}^{3}}$ and $\bar{w}$ in $k^{*}\left(\operatorname{Spin}(n) ; \mathbf{Z}_{2}\right) / \operatorname{kerj}^{*}$ such that $\bar{n}^{*}\left(\bar{w}_{i}\right)=\bar{x}_{i}, \bar{n}^{*}\left(\bar{u}_{j}\right)=\bar{y}_{j}, \bar{n}^{*}\left(\bar{v}_{k}\right)=$ $\bar{z}_{k}$ and $\bar{n}^{*}(\bar{w})=\bar{x}$. These elements are uniquely determined modulo $I \mathrm{~mm}^{*}$. Furthermore all of them have zero square since the square of the elements of odd degree in $K^{*}\left(\operatorname{Spin}(n) ; \mathbf{Z}_{2}\right)$ is zero [3].

Therefore there exists an algebra homomorphism

$$
g: \Lambda_{\mathbf{z}_{2}\{t-1]}\left(\left(\bar{w}_{i}\right)_{i \in S_{n}^{1}},\left(\bar{u}_{j}\right)_{j \in S_{n}^{2}},\left\langle\bar{v}_{k}\right)_{k \in S_{n}^{s}}, \bar{w}\right) \longrightarrow k^{*}\left(\operatorname{Spin}(n) ; \mathbf{Z}_{2}\right)
$$

It is easy to verify that $g$ is an isomorphism.

We have therefore the following theorem.

\subsection{Theorem.}

The $\mathbf{Z}_{2}\left[t^{-1}\right]$ algebra $k^{*}\left(\operatorname{Spin}(n) ; \mathbf{Z}_{2}\right) /$ kerj $j^{*}$ is an exterior algebra generated by $\left(\bar{w}_{i}\right)_{i \in S_{n}^{2}},\left(\bar{u}_{j}\right)_{j \in S_{n}^{2}},\left(\bar{v}_{k}\right)_{k \in S_{n}^{3}}$ and $\bar{u}$.

The next proposition is an immediate consequence of proposition 2.2 .

\subsection{Proposition.}

The torsion coefficients of $k^{*}(\operatorname{Spin}(n))$ are two and for all $y \in k^{*}(\operatorname{Spin}(n)) 2 y=$ 0 if and only if $t^{-1} y=0$.

Acknowledgement: I would like to thank Dr. Alan Robinson for some interesting comments during a visit by the author to Warwick University.

\section{References}

1. M.F. AtiYA AN F. Hirzebruch, Vector bundles and homogeneous spaces, Proc. Symp. Pure Math. A.M.S. 3 (1961), 7-38.

2. A. BOREL, Topology of Lie groups and characteristic classes, Bull. Am. Math. Soc. 61 (1955), 397-432.

3. L. HoDGKIN, On the $K$-theory of Lie groups, Topology 6 (1967), 1-36.

4. L. MAGALHÃES, Some results on the connective $K$-theory of Lie groups, Bull. Can. Math. Soc. 31. (1988), 194-199.

5. J.P. MAY, " $E_{\infty}$ ring spaces and $E_{\infty}$ ring spectra," Lecture Notes in Mathematics 577, Springer-Verlag, 1977.

Keywords. connective $K$-theory, Spinor groups, Atiyah-Hirzebruch spectral sequence 1980 Mathematics subject classifications: $55 N 15,55 N 20$

Centro de Matemática

Faculdade de Ciéncias

Universidade do Porto

PORTUGAL

Rebut el 28 de Març de 1989 